\title{
Verschwindungssätze, die bei der Berechnung der Dimension des Vektorraums der Spitzenformen zur Modulgruppe $n$-ten Grades und Stufe $q>2$ auftreten
}

von

\author{
Petra Ploch (Freiburg i. Br.)
}

1. Problemstellung. Ein wichtiges ungelöstes Problem aus der Theorie der Siegelschen Modulformen ist die Berechnung der Dimension des Vektorraums der Spitzenformen zur Modulgruppe $n$-ten Grades und Stufe $q, \Gamma(n, q)$. Nach [3] wird diese gegeben durch

$$
\sigma(\Gamma(n, q), g)=\eta(n, g) \int_{\mathfrak{F}} \sum_{M \in \Gamma(n, q)} R(M, Z) d w_{Z} .
$$

Hierbei bezeichnet $g$ das Gewicht der Spitzenformen, wobei man aus Konvergenzgründen $g>2 n$ voraussetzt, $\mathfrak{F}$ ist ein Fundamentalbereich von $\Gamma(n, q)$ in der Siegelschen oberen Halbebene $n$-ten Grades $\mathfrak{Z}(n), \eta(n, g)$ wurde in [3], (19), definiert und $d w_{Z}$ ist das symplektische Volumenelement. Für $M=\left(\begin{array}{ll}A & B \\ C & D\end{array}\right) \in \Gamma(n, q)$ mit $n \times n$ Matrizen $A, B, C$ und $D$, sowie für $Z=X+i Y \in \mathfrak{Z}(n)$ setzt man weiter

$$
M\langle Z\rangle:=(A Z+B)(C Z+D)^{-1}, \quad M\{Z\}:=C Z+D
$$

und

$$
R(M, Z):=\operatorname{det}(-\bar{Z}+M\langle Z\rangle)^{-g} \operatorname{det}(M\{Z\})^{-g}(\operatorname{det} Y)^{g} .
$$

Will man dieses Integral mit Hilfe der Selbergschen Spurformel, die in [15] beschrieben wird, auswerten, so zerlegt man zunächst $\Gamma(n, q)$ in endlich viele, paarweise disjunkte, konjugationsinvariante Teilmengen und betrachtet dann die Einzelintegrale. Diese Teilmengen hängen mit den charakteristischen Polynomen der betrachteten Matrizen zusammen. Man vermutet, daß Matrizen mit einem charakteristischen Polynom $\chi(M, x) \neq(x-1)^{2 n}$ keinen Beitrag zum Dimensionsintegral liefern. In [14] wurde das Verschwinden des Dimensionsintegrals für Matrizen mit einem über $\mathbb{Q}$ irreduziblen charakteristischen Polynom gezeigt. In der vorliegenden Arbeit sollen die Fälle behandelt werden, bei denen man das Verschwinden des Dimensions- 
integrals durch Einfügen von konvergenzerzeugenden Faktoren zeigen kann. Genauer soll gezeigt werden:

Theorem. Es sei $q>2$,

$K_{0}:=\{M \in \Gamma(n, q) \mid \chi(M, x)$ ist reduzibel über $\mathbb{Q}$ und es gibt einen Eigenwert e von $M$ mit $|e| \neq 1$, derart, daß für alle möglichen Zerlegungen $\chi(M, x)=g(x) h(x) h^{*}(x)$ über $\mathbb{Q}$ mit $h(1 / x) x^{j}= \pm h^{*}(x)$ stets $h(e) \neq 0$ gilt $\}$,

$K_{00}:=\left\{M \in \Gamma(n, q) \mid\right.$ Ist $M \in \Gamma_{i}(n), R \in \Gamma(n)$ und $R M R^{-1} \in \Gamma_{i}(n)$, so folgt $\left.R \in \Gamma_{i}(n)\right\}$

und $K:=K_{0} \cap K_{00}$. Dann folgt

$$
\int_{\mathfrak{F}} \sum_{M \in K} R(M, Z) d w_{Z}=0 .
$$

2. Bezeichnungen und grundlegende Bemerkungen. Die symplektische Gruppe $n$-ten Grades $\Sigma(n)$ besteht aus allen reellen $2 n \times 2 n$ Matrizen $M$ mit $M^{\prime} I_{n} M=I_{n}$. Hierbei sei $M^{\prime}$ die Transponierte von $M$ und mit $I_{n}$ bezeichnet man die symplektische Einheitsmatrix n-ten Grades, d.h.

$$
I_{n}:=\left(\begin{array}{rr}
0_{n} & E_{n} \\
-E_{n} & 0_{n}
\end{array}\right),
$$

wobei $E_{n}$ bzw. $0_{n}$ die $n \times n$ Einheits- bzw. Nullmatrix ist. Die Siegelsche Modulgruppe $n$-ten Grades $\Gamma(n)$ besteht aus allen symplektischen Matrizen mit ganzzahligen Koeffizienten und für $q \in \mathbb{N}$ wird durch $\Gamma(n, q):=\{M \in$ $\left.\Gamma(n) \mid M \equiv E_{2 n} \bmod q\right\}$ die Hauptkongruenzgruppe $q$-ter Stufe von $\Gamma(n)$ definiert.

Für $i \in \mathbb{N}$ mit $1 \leq i \leq n$ besteht die $i$-te Spitzengruppe $\Sigma_{i}(n)$ aus allen $M \in \Sigma(n)$ der Gestalt

$$
M=\left(\begin{array}{llll}
A & 0 & B & * \\
* & P^{\prime} & * & * \\
C & 0 & D & * \\
0 & 0 & 0 & P^{-1}
\end{array}\right)
$$

mit $(n-i) \times(n-i)$ Matrizen $A, B, C$ und $D$, sowie einer $i \times i$ Matrix $P$. Analog definiert man $\Gamma_{i}(n)$ und $\Gamma_{i}(n, q)$.

Es sei $n=n_{1}+\ldots+n_{r}$ mit $n_{i} \in \mathbb{N}(i=1, \ldots, r) . \Sigma\left(n_{1}, \ldots, n_{r}\right)$ besteht aus allen Matrizen der Gestalt

$$
M=\left(\begin{array}{ll}
A & B \\
C & D
\end{array}\right)=:\left\{M_{1}, \ldots, M_{r}\right\} \in \Sigma(n)
$$

mit 


$$
\begin{array}{ll}
A=\left(\begin{array}{lll}
A_{1} & & 0 \\
& \ddots & \\
0 & & A_{r}
\end{array}\right), \quad B=\left(\begin{array}{lll}
B_{1} & & 0 \\
& \ddots & \\
0 & & B_{r}
\end{array}\right), \\
C=\left(\begin{array}{lll}
C_{1} & & 0 \\
& \ddots & \\
0 & & C_{r}
\end{array}\right), \quad D=\left(\begin{array}{ccc}
D_{1} & & 0 \\
& \ddots & \\
0 & & D_{r}
\end{array}\right)
\end{array}
$$

und

$$
M_{i}:=\left(\begin{array}{cc}
A_{i} & B_{i} \\
C_{i} & D_{i}
\end{array}\right) \in \Sigma\left(n_{i}\right) \quad(i=1, \ldots, r) .
$$

Es sei $\mu$ eine Untergruppe von $\Sigma(n)$. $Z$ heißt ein elliptischer Fixpunkt von $\mu$, wenn es ein $M \in R \backslash\left\{ \pm E_{2 n}\right\}$ mit $M\langle Z\rangle=Z$ gibt. [4] kann man entnehmen, daß $\Gamma(n, q)$ für $q>2$ keine elliptischen Fixpunkte besitzt.

Es sei $\mu$ eine Untergruppe von $\Sigma(n) . M, N \in \mu$ heißen konjugiert in $\mu$, falls es ein $L \in \mu$ mit $M=L^{-1} N L$ gibt. Die Menge

$$
\{M\}_{\mu}:=\left\{N \in \mu \mid \text { Es gibt ein } L \in \mu \text { mit } M=L^{-1} N L\right\}
$$

heißt die Konjugationsklasse von $M$ in $\mu$. Das charakteristische Polynom einer Matrix und $\operatorname{Rang}(M-a E)^{b}$ mit $a \in \mathbb{C}$ und $b \in \mathbb{N}$ sind invariant unter Konjugation.

Es sei $Y$ eine reelle symmetrische $n \times n$ Matrix. $Y$ heißt positiv, $Y>0$, wenn $Y[x]:=x^{\prime} Y x>0$ für alle reellen $n$-reihigen Spalten $x \neq 0$ ist. Die Menge der positiven $n \times n$ Matrizen sei $\mathfrak{Y}(n)$. Zerlegt man $Y$ in der Gestalt

$$
Y=\left(\begin{array}{ll}
Y_{1} & Y_{12} \\
Y_{12}^{\prime} & Y_{2}
\end{array}\right)
$$

mit einer $r \times r$ Matrix $Y_{1}$, einer $r \times(n-r)$ Matrix $Y_{12}$ und einer $(n-r) \times(n-r)$ Matrix $Y_{2}$, so gilt

$$
Y=\left(\begin{array}{cc}
P_{1} & 0 \\
0 & P_{2}
\end{array}\right)\left[\left(\begin{array}{cc}
E & L \\
0 & E
\end{array}\right)\right]
$$

mit $P_{1}=Y_{1}, L=Y_{1}^{-1} Y_{12}, P_{2}=Y_{2}-Y_{1}^{-1}\left[Y_{12}\right]$.

3. Das charakteristische Polynom einer Matrix $M \in \Gamma(n, q)$ mit $q>2$. Wie in [14] folgt aus [1]:

Satz 1. Es sei $M \in \Gamma(n, q)$ mit $q>2$. Dann zerfällt das charakteristische Polynom von $M, \chi(M, x)$, über $\mathbb{C}$, in der folgenden Weise:

(3) $\chi(M, x)=(x-1)^{e} p_{1}^{s_{1}}(x) \ldots p_{k}^{s_{k}}(x) v_{1}^{t_{1}}(x) \ldots v_{m}^{t_{m}}(x) w_{1}^{r_{1}}(x) \ldots w_{j}^{r_{j}}(x)$ mit

$$
\begin{gathered}
e \equiv 0 \bmod 2, \\
p_{i}(x)=(x-b)\left(x-b^{-1}\right), \quad b \in \mathbb{R} \backslash \mathbb{Q}(i=1, \ldots, k),
\end{gathered}
$$


$v_{i}(x)=(x-a)(x-\bar{a}), \quad a \bar{a}=1, a \notin \mathbb{R}(i=1, \ldots, m)$,
$w_{i}(x)=(x-g)(x-\bar{g})\left(x-g^{-1}\right)\left(x-\bar{g}^{-1}\right), \quad g \notin \mathbb{R}, g \bar{g} \neq 1(i=1, \ldots, j)$.

Haben alle Eigenwerte von $M$ den Absolutbetrag 1, so gilt

$$
\chi(M, x)=(x-1)^{2 n} .
$$

Die vorliegende Arbeit beschäftigt sich nur mit Matrizen aus $K$. Betrachtet man also alle möglichen Zerlegungen des charakteristischen Polynoms $\chi(M, x)$ einer Matrix $M \in K$ über $\mathbb{Q}$ der Form $\chi(M, x)=g(x) h(x) h^{*}(x)$ mit $x^{j} h(1 / x)= \pm h^{*}(x)$, so kann man im folgenden annehmen, daß $g(x)$ entweder einen Faktor der Gestalt $p(x)$ oder $w(x)$ enthält und daß dieser Faktor nicht in $h(x)$ oder $h^{*}(x)$ auftritt. Man setze:

$$
\begin{aligned}
& K_{1 j}:=\left\{M \in K \mid \text { Es gibt ein } b \in \mathbb{R} \backslash \mathbb{Q} \text { mit } \chi(M, x)=(x-b)^{j} f(x)\right. \\
& K_{2 j}:=\left\{M \in K \backslash \sum_{j=1}^{n} K_{1 j} \mid \text { Es gibt ein } g \notin \mathbb{R} \text { mit } g \bar{g} \neq 1\right. \text { und }
\end{aligned}
$$

Trivialerweise ist

$$
K=\sum_{j=1}^{n} K_{1 j} \cup \sum_{j=1}^{[n / 2]} K_{2 j}
$$

eine disjunkte Zerlegung von $K$ und $K_{1 j}(j=1, \ldots, n)$, sowie $K_{2 j}(j=$ $1, \ldots,[n / 2])$ sind jeweils invariant unter Konjugation.

Will man die Beiträge von $K_{i j}$ zum Dimensionsintegral mit Hilfe der Selbergschen Spurformel berechnen, so muß man in den betreffenden Einzelintegralen zunächst Summation und Integration vertauschen. Dies ist ohne konvergenzerzeugende Faktoren im allgemeinen nicht möglich.

4. Die konvergenzerzeugenden Faktoren. Es sei $L$ eine konjugationsinvariante Teilmenge von $K$ und $l$ der Beitrag von $L$ zum Dimensionsintegral, so ergibt sich, wie in [14],

$$
l=p \int_{\mathfrak{F}} \sum_{M \in L} R(M, Z) d w_{Z},
$$


mit einer Konstanten $p$, und $\mathfrak{F}$ ist ein Fundamentalbereich von $\Gamma(n)$ in $\mathfrak{Z}(n)$. Benötigt man also bei der Vertauschung von Summation und Integration konvergenzerzeugende Faktoren, so kann man sich auf die Spitze bei Unendlich beschränken.

Über die Vertauschbarkeit von Summation und Integration kann man folgendes aussagen. Es sei $\mathfrak{X}$ die Menge der reellen symmetrischen $n \times n$ Matrizen $X=\left(x_{i j}\right)_{i, j=1}^{n}$ mit $\left|x_{i j}\right| \leq 1 / 2(i, j=1, \ldots, n)$ und $t \subset \Gamma(n)$. Dann setze man

$$
T(t, g, Y):=\int_{\mathfrak{X}} \sum_{t} R(M, Z) d X .
$$

Nach [7] gilt

$$
T(t, g, Y) \prec y_{1}^{a_{1}} \ldots y_{n}^{a_{n}} .
$$

Hierbei bedeutet die Schreibweise $f(x) \prec g(x)$, daß es eine Konstante $c>0$ gibt mit $f(x) \leq c g(x)$ für alle $x \in D$, dem Bereich in dem $f(x)$ und $g(x)$ definiert sind. Weiter bezeichnen $y_{1}, \ldots, y_{n}$ die Diagonalelemente von $Y$ und für die Koeffizienten $a_{1}, \ldots, a_{n}$ gilt

$$
a_{r}+\ldots+a_{n} \leq r+\ldots+n \quad(r=1, \ldots, n) .
$$

Nach [8] kann man Summation und Integration vertauschen, wenn

$$
a_{r}+\ldots+a_{n}<r+\ldots+n \quad(r=1, \ldots, n)
$$

gilt. Will man dies nachprüfen, so muß man untersuchen, ob es Elemente in einer Spitzengruppe gibt, denn aus $t \cap \Gamma_{n-k+1}=\emptyset$ folgt $a_{k}+\ldots+a_{n}<$ $k+\ldots+n$.

Ob es Elemente in einer Spitzengruppe gibt, kann man aus dem charakteristischen Polynom ablesen. [10] kann man entnehmen, daß es bis auf die Faktoren $(x-a)(x-\bar{a})$, mit $a \notin \mathbb{R}$ und $a \bar{a}=1$, genau dann eine zu $M$ $\Gamma(n)$-konjugierte Matrix in $\Gamma_{k}(n)$ gibt, wenn das charakteristische Polynom über $\mathbb{Q}$ in der Gestalt

$$
\chi(M, x)=f(x) h(x) h^{*}(x)
$$

mit

$$
\begin{gathered}
\operatorname{grad} f(x)=2(n-k), \quad \operatorname{grad} h(x)=\operatorname{grad} h^{*}(x)=k, \\
f(x)=x^{2(n-k)} f(1 / x), \quad h^{*}(x)=d x^{k} h(1 / x), \quad d \in \mathbb{Q} \backslash\{0\},
\end{gathered}
$$

aufspaltet. Da es in $K_{1 j}(j=1, \ldots, n)$ und $K_{2 j}(j=1, \ldots,[n / 2])$ Elemente in Spitzengruppen geben kann, ist eine Vertauschung von Summation und Integration im Dimensionsintegral im allgemeinen nicht möglich.

Man konstruiert jetzt die konvergenzerzeugenden Faktoren.

SATZ 2. Übt man auf $Y \in \mathfrak{Y}(n)$ eine Jacobische Transformation

$$
Y=\left(\begin{array}{cc}
P_{1} & 0 \\
0 & P_{2}
\end{array}\right)\left[\left(\begin{array}{cc}
E & L \\
0 & E
\end{array}\right)\right]
$$


mit einer $(n-r) \times(n-r)$ Matrix $P_{1}$, einer $r \times r$ Matrix $P_{2}$ und einer $(n-r) \times r$ Matrix L aus, so gibt es eine Konstante $c>0$ mit

$$
c y_{n-r+1} \ldots y_{n} \leq \operatorname{det} P_{2} \leq y_{n-r+1} \ldots y_{n} .
$$

B ew e is. Für $Y \in \mathfrak{Y}(n)$ gibt es eine Darstellung $Y=Q[D]$ mit

$$
Q=\left(\begin{array}{lll}
q_{1} & & 0 \\
& \ddots & \\
0 & & q_{n}
\end{array}\right)=:\left(\begin{array}{ll}
Q_{1} & 0 \\
0 & Q_{2}
\end{array}\right)
$$

und

$$
D=\left(\begin{array}{lll}
1 & & * \\
& \ddots & \\
0 & & 1
\end{array}\right)=:\left(\begin{array}{ll}
D_{1} & D_{12} \\
0 & D_{2}
\end{array}\right)
$$

mit $(n-r) \times(n-r)$ Matrizen $Q_{1}$ und $D_{1}, r \times r$ Matrizen $Q_{2}$ und $D_{2}$, sowie einer $(n-r) \times r$ Matrix $D_{12}$. Durch einen Koeffizientenvergleich erhält man $P_{2}=D_{2}^{\prime} Q_{2} D_{2}$. Aus $\operatorname{det} D_{2}=1$ und $\operatorname{det} Q_{2}=q_{n-r+1} \ldots q_{n}$ erhält man $\operatorname{det} P_{2}=q_{n-r+1} \ldots q_{n}$. Nach [4] gibt es positive Konstanten $c_{n-r+1}, \ldots, c_{n}$ mit $1 \leq y_{j} / q_{j} \leq c_{j}(j=n-r+1, \ldots, n)$. Folglich gilt

$$
c y_{n-r+1} \ldots y_{n} \leq \operatorname{det} P_{2} \leq y_{n-r+1} \ldots y_{n}
$$

mit $c:=\left(c_{n-r+1} \ldots c_{n}\right)^{-1}$.

Durch Einfügen dieser Faktoren kann man auf Grund der folgenden Überlegungen tatsächlich Summation und Integration vertauschen.

Es sei $G$ eine Menge mit

$$
G \cap \Gamma_{\mu}(n) \neq \emptyset, \quad G \cap \Gamma_{j}(n)=\emptyset \quad(j=\mu+1, \ldots, n) .
$$

Dann erhält man im Dimensionsintegral

$$
\begin{array}{ll}
a_{k}+\ldots+a_{n}<k+\ldots+n & (k=1, \ldots, n-\mu), \\
a_{k}+\ldots+a_{n} \leq k+\ldots+n & (k=n-\mu+1, \ldots, n) .
\end{array}
$$

Fügt man also den Faktor $\left(\operatorname{det} P_{2}\right)^{-e}$ mit einer $\mu \times \mu$ Matrix $P_{2}$ ein, so erhält man jetzt in dem betreffenden Integral

$$
a_{k}+\ldots+a_{n}<k+\ldots+n \quad(k=1, \ldots, n)
$$

und man kann damit Summation und Integration vertauschen.

Die konvergenzerzeugenden Faktoren müssen bei den weiteren Konjugationen invariant bleiben. Man muß sich jetzt also überlegen, wann dies der Fall ist.

SATZ 3. Es sei

$$
Y=\left(\begin{array}{ll}
P_{1} & 0 \\
0 & P_{2}
\end{array}\right)\left[\left(\begin{array}{ll}
E & L \\
0 & E
\end{array}\right)\right] \in \mathfrak{Y}(n)
$$


mit einer $(n-r) \times(n-r)$ Matrix $P_{1}$, einer $r \times r$ Matrix $P_{2}$ und einer $(n-r) \times r$ Matrix L. Dann ist $\operatorname{det} P_{2}$ invariant unter der Transformation $X+i Y \rightarrow M\langle X+i Y\rangle$ mit

$$
M=\left(\begin{array}{llll}
A & 0 & B & * \\
* & T^{\prime} & * & * \\
C & 0 & D & D_{1} \\
0 & 0 & 0 & T^{-1}
\end{array}\right) \in \Sigma_{r}(n)
$$

mit $\operatorname{det} T= \pm 1$.

B ew eis. Es seien $M$ und $Y$ in der obigen Gestalt gegeben und

$$
M\langle Z\rangle=: X_{M}+i Y_{M}=: X_{M}+i\left(\begin{array}{cc}
\underline{P}_{1} & 0 \\
0 & \underline{P}_{2}
\end{array}\right)\left[\left(\begin{array}{cc}
E & \underline{L} \\
0 & E
\end{array}\right)\right] .
$$

Nach [4] gilt $Y_{M}=(M\{Z\})^{\prime-1} Y \overline{(M\{Z\})}^{-1}$. Setzt man weiter

$$
X=\left(\begin{array}{ll}
X_{1} & X_{12} \\
X_{12}^{\prime} & X_{2}
\end{array}\right)
$$

mit einer $(n-r) \times(n-r)$ Matrix $X_{1}$, einer $(n-r) \times r$ Matrix $X_{12}$ und einer $r \times r$ Matrix $X_{2}$, so erhält man durch einen Koeffizientenvergleich

$$
\begin{aligned}
\underline{P}_{1} & =\left(C\left(X_{1}+i P_{1}\right)+D\right)^{\prime-1} P_{1} \overline{\left(C\left(X_{1}+i P_{1}\right)+D\right)}-1 \\
\underline{L} & =\overline{\left(C\left(X_{1}+i P_{1}\right)+D\right)} L T-\overline{\left(C\left(X_{12}+i P_{1} L\right)+D_{1}\right)} T, \\
\underline{P}_{2} & =T^{\prime} P_{2} T .
\end{aligned}
$$

Hieraus folgt trivialerweise $\operatorname{det} P_{2}=\operatorname{det} \underline{P}_{2}$.

5. Beweis des Theorems für $K_{1 j}(j=1, \ldots, n)$. Für die Matrizen $M \in K_{1 j}$ gilt

$$
M=\left(\begin{array}{llll}
A & 0 & B & * \\
* & P^{\prime} & * & * \\
C & 0 & D & * \\
0 & 0 & 0 & P^{-1}
\end{array}\right)
$$

mit $(n-k) \times(n-k)$ Matrizen $A, B, C$ und $D$, sowie einer $k \times k$ Matrix $P$. Hierbei gilt $n-k \geq j$ und es gibt einen Eigenwert $b \in \mathbb{R} \backslash \mathbb{Q}$ von $M$ mit $(x-b) \nmid \chi(P, x)$ und $(x-b) \nmid \chi\left(P^{-1}, x\right)$.

Im Dimensionsintegral fügt man jetzt für die Matrizen aus $\Gamma_{i}(n) \backslash$ $\bigcup_{j=i+1}^{n} \Gamma_{j}(n)$ den Faktor $\left(\operatorname{det} P_{2}\right)^{-e}$ mit einer $i \times i$ Matrix $P_{2}$ ein, wobei $P_{2}$ der entsprechende Anteil aus der Jacobischen Transformation von $\operatorname{Im} Z$ ist. Nach Satz 2 kann man dann Summation und Integration vertauschen und die Selbergsche Spurformel anwenden.

Im folgenden konjugiert man die Matrizen mit reellen symplektischen Matrizen auf die gleiche Normalform, die man in [14] für die Matrizen aus 
$K_{j}$ erhalten hat. Hierbei muß man darauf achten, daß die konvergenzerzeugenden Faktoren invariant bleiben.

Es sei also

$$
M=\left(\begin{array}{llll}
A & 0 & B & * \\
* & P^{\prime} & * & * \\
C & 0 & D & * \\
0 & 0 & 0 & P^{-1}
\end{array}\right) \in K_{1 j} \cap \Gamma_{i}(n) \backslash \bigcup_{k=i+1}^{n} \Gamma_{k}(n) .
$$

Konjugiert man $M$ mit einer Matrix

$$
R=\left\{\left(\begin{array}{cc}
\underline{A} & \underline{B} \\
\underline{C} & \underline{D}
\end{array}\right),\left(\begin{array}{cc}
E & 0 \\
0 & E
\end{array}\right)\right\} \in \Sigma(n-i, i),
$$

so erhält man eine Matrix der Gestalt

$$
M=\left(\begin{array}{llllll}
K^{\prime} & 0 & 0 & 0 & 0 & T \\
0 & A_{1} & 0 & 0 & B_{1} & * \\
S & * & P^{\prime} & T^{\prime} & * & * \\
0 & 0 & 0 & K^{-1} & 0 & * \\
0 & C_{1} & 0 & 0 & D_{1} & * \\
0 & 0 & 0 & 0 & 0 & P^{-1}
\end{array}\right)
$$

mit

$$
K=\left(\begin{array}{cccc}
b & * & & 0 \\
& \ddots & \ddots & \\
& & & * \\
0 & & & b
\end{array}\right) \quad \text { und } \quad(x-b) \nmid \chi\left(\left(\begin{array}{cc}
A_{1} & B_{1} \\
C_{1} & D_{1}
\end{array}\right), x\right) .
$$

Konjugiert man diese Matrix mit einer Matrix der Gestalt

$$
R=\left(\begin{array}{cccccc}
E & 0 & 0 & & & \\
0 & E & 0 & & 0 & \\
V & 0 & E & & & \\
& & & E & 0 & -V^{\prime} \\
& 0 & & 0 & E & 0 \\
& & & 0 & 0 & E
\end{array}\right) \in \Sigma_{i}(n),
$$

so erhält man

$$
M=\left(\begin{array}{llllll}
K^{\prime} & 0 & 0 & 0 & 0 & * \\
0 & A_{1} & 0 & 0 & B_{1} & * \\
X & * & P^{\prime} & * & * & * \\
0 & 0 & 0 & K^{-1} & * & * \\
0 & C_{1} & 0 & 0 & D_{1} & * \\
0 & 0 & 0 & 0 & 0 & P^{-1}
\end{array}\right)
$$

mit

$$
X=V K^{\prime}-P^{\prime} V+S .
$$


Betrachtet man die Gleichung $V K^{\prime}-P^{\prime} V=-S$ als lineares Gleichungssystem für die Koeffizienten von $V$, so kann man also genau dann stets $X=0$ erreichen, wenn die Koeffizientenmatrix $W$ nicht singulär ist. Da $\operatorname{det} W=$ $\left|\chi\left(P^{\prime}, b\right)\right|$ gilt und $b$ kein Eigenwert von $P^{\prime}$ ist, ist dies immer möglich. Da $M$ symplektisch ist, gibt es also stets eine konjugierte Matrix

$$
M=\left(\begin{array}{llllll}
K^{\prime} & 0 & 0 & 0 & 0 & X \\
0 & A_{1} & 0 & 0 & B_{1} & * \\
0 & * & P^{\prime} & * & * & * \\
0 & 0 & 0 & K^{-1} & 0 & 0 \\
0 & C_{1} & 0 & 0 & D_{1} & * \\
0 & 0 & 0 & 0 & 0 & P^{-1}
\end{array}\right) .
$$

Bei den hierbei verwendeten Transformationen bleibt der konvergenzerzeugende Faktor invariant. Durch analoge Überlegungen kann man diese Matrix durch Konjugation mit einer Matrix der Gestalt

$$
R=\left(\begin{array}{cc}
E & S \\
0 & E
\end{array}\right) \quad \text { mit } \quad S=\left(\begin{array}{lll}
0 & 0 & V \\
0 & 0 & 0 \\
V^{\prime} & 0 & 0
\end{array}\right)
$$

auf die Gestalt

$$
M=\left\{\left(\begin{array}{ll}
K^{\prime} & 0 \\
0 & K^{-1}
\end{array}\right),\left(\begin{array}{llll}
A_{1} & 0 & B_{1} & * \\
* & P^{\prime} & * & * \\
C_{1} & 0 & D_{1} & * \\
0 & 0 & 0 & P^{-1}
\end{array}\right)\right\}
$$

bringen, wobei nach Satz 3 der konvergenzerzeugende Faktor invariant bleibt. Insgesamt ist damit gezeigt:

SATZ 4. Es sei

$$
M \in K_{1 j} \cap \Gamma_{i}(n) \backslash \bigcup_{k=i+1}^{n} \Gamma_{k}(n) \quad(j=1, \ldots, n, i \leq n-j) .
$$

Dann kann man $M$ durch Transformationen, die $\operatorname{det} P_{2}$ mit einer $i \times i$ Matrix $P_{2}$ invariant lassen, auf die Gestalt

$$
M=\left\{\left(\begin{array}{ll}
K^{\prime} & 0 \\
0 & K^{-1}
\end{array}\right),\left(\begin{array}{ll}
A & B \\
C & D
\end{array}\right)\right\}
$$

mit

$$
K=\left(\begin{array}{cccc}
b & * & & 0 \\
& \ddots & \ddots & \\
& & & * \\
0 & & & b
\end{array}\right) \quad \text { und } \quad(x-b) \nmid \chi\left(\left(\begin{array}{cc}
A & B \\
C & D
\end{array}\right), x\right)
$$

bringen. 
Bis auf die Reihenfolge der Kästchen entspricht diese Matrix gerade der Matrix (10) aus [14]. Das Dimensionsintegral entspricht also demjenigen der Matrizen mit einem über $\mathbb{Q}$ irreduziblen charakteristischen Polynom und einem Eigenwert $b \in \mathbb{R} \backslash \mathbb{Q}$ bis auf die konvergenzerzeugenden Faktoren. Da man das Verschwinden des Dimensionsintegrals in [14] unabhängig von $\operatorname{Im} Z$ gezeigt hat, folgt, daß die Matrizen aus $K_{1 j}(j=1, \ldots, n)$ keinen Beitrag zum Dimensionsintegral liefern.

6. Beweis des Theorems für $K_{2 j}(j=1, \ldots,[n / 2])$. Für die Matrizen $M \in K_{2 j}(j=1, \ldots,[n / 2])$ gilt

$$
M=\left(\begin{array}{llll}
A & 0 & B & * \\
* & P^{\prime} & * & * \\
C & 0 & D & * \\
0 & 0 & 0 & P^{-1}
\end{array}\right)
$$

mit $(n-k) \times(n-k)$ Matrizen $A, B, C$ und $D$, sowie einer $k \times k$ Matrix $P$. Hierbei gilt $n-k \geq 2 j$ und es gibt einen Eigenwert $g \notin \mathbb{R}$ mit $g \bar{g} \neq 1$, $(x-g) \nmid \chi(P, x),(x-\bar{g}) \nmid \chi(P, x),\left(x-g^{-1}\right) \nmid \chi(P, x)$ und $\left(x-\bar{g}^{-1}\right) \nmid \chi(P, x)$.

Wieder fügt man für die Matrizen aus $\Gamma_{i}(n) \backslash \bigcup_{k=i+1}^{n} \Gamma_{k}(n)$ den entsprechenden konvergenzerzeugenden Faktor $\left(\operatorname{det} P_{2}\right)^{-e}$ ein und kann damit Summation und Integration vertauschen. Durch Konjugation von $M \in K_{2 j} \cap$ $\Gamma_{i}(n) \backslash \bigcup_{k=i+1}^{n} \Gamma_{k}(n)$ mit einer Matrix $R \in \Gamma_{i}(n)$ kann man

$$
M=\left(\begin{array}{llllll}
V^{\prime} & 0 & 0 & 0 & 0 & * \\
0 & A_{1} & 0 & 0 & B_{1} & * \\
* & * & P^{\prime} & * & * & * \\
0 & 0 & 0 & V^{-1} & 0 & * \\
0 & C_{1} & 0 & 0 & D_{1} & * \\
0 & 0 & 0 & 0 & 0 & P^{-1}
\end{array}\right)
$$

mit

$$
\chi(V, x)=(x-g)^{j}(x-\bar{g})^{j} \quad \text { und } \quad(x-g) \nmid \chi\left(\left(\begin{array}{cc}
A_{1} & B_{1} \\
C_{1} & D_{1}
\end{array}\right), x\right)
$$

erreichen. Hierbei bleibt nach Satz $3 \operatorname{det} P_{2}$ invariant. Da $g, \bar{g}, g^{-1}$ und $\bar{g}^{-1}$ keine Eigenwerte von $P$ sind, kann man auch hier folgendes erreichen:

SATZ 5. Es sei

$$
M \in K_{2 j} \cap \Gamma_{i}(n) \backslash \bigcup_{k=i+1}^{n} \Gamma_{k}(n) \quad(j=1, \ldots,[n / 2], n-k \geq 2 j) .
$$

Dann kann man $M$ durch Transformationen, die $\operatorname{det} P_{2}$, mit einer $i \times i$ Matrix $P_{2}$, invariant lassen, auf die Gestalt 


$$
M=\left\{\left(\begin{array}{ll}
V^{\prime} & 0 \\
0 & V^{-1}
\end{array}\right),\left(\begin{array}{ll}
A & B \\
C & D
\end{array}\right)\right\}
$$

bringen. Hierbei gilt

$$
\chi(V, x)=(x-g)^{j}(x-\bar{g})^{j} \quad \text { und } \quad(x-g) \nmid \chi\left(\left(\begin{array}{cc}
A & B \\
C & D
\end{array}\right), x\right) .
$$

Auch hier entspricht die Matrix (8) gerade der Matrix (11) aus [14] bis auf die Reihenfolge der Kästchen. Mit den gleichen Argumentationen, wie im Fall $K_{1}$, folgt auch hier das Verschwinden des Dimensionsintegrals.

Damit ist das Theorem bewiesen.

\section{Literaturverzeichnis}

[1] U. Christian, A reduction theory for symplectic matrices, Math. Z. 101 (1967), 213-244.

[2] - Berechnung des Ranges der Schar der Spitzenformen zur Modulgruppe zweiten Grades und Stufe $q>2$, J. Reine Angew. Math. 277 (1975), 130-154.

[3] —, Siegelsche Modulformen und Integralgleichungen, Math. Z. 101 (1967), 299-305.

[4] -, Siegelsche Modulfunktionen, Vorlesungsausarbeitung, Math. Inst. Univ. Göttingen, 1974/75.

[5] - Über die Anzahl der Spitzen Siegelscher Modulgruppen, Abh. Math. Sem. Univ. Hamburg 32 (1968), 55-60.

[6] -, Über elliptische Fixpunkte symplektischer Matrizen, Monatsh. Math. 72 (1968), 289-295.

[7] -, Untersuchung einer Poincaréschen Reihe I, J. Reine Angew. Math. 233 (1968), 37-88.

[8] -, Untersuchung einer Poincaréschen Reihe II, ibid. 237 (1969), 12-25.

[9] —, Zur Berechnung des Ranges der Schar der Spitzenformen zur Modulgruppe zweiten Grades und Stufe $q>2$, ibid. 296 (1977), 108-118.

[10] -, Zur Theorie der symplektischen Gruppen, Acta Arith. 24 (1973), 61-85.

[11] K. Hashimoto, The dimension of the space of cusp forms on Siegel upper half plane of degree two I, J. Fac. Sci. Univ. Tokyo Sect. IA Math. 30 (1983), 403-488.

[12] - The dimension of the space of cusp forms on Siegel upper half plane of degree two. II: The $\mathbb{Q}$-range one case, Math. Ann. 266 (1984), 539-559.

[13] Y. Morita, An explicit formula for the dimension of spaces of Siegel modular forms of degree II, J. Fac. Sci. Univ. Tokyo 21 (1944), 167-248.

[14] P. Ploch, Matrizen mit einem über $\boldsymbol{Q}$ irreduziblen charakteristischen Polynom und die Dimension des Vektorraums der Spitzenformen zur Modulgruppe n-ten Grades und Stufe $q>2$, Acta Arith. 57 (1991), 257-266.

[15] A. Selberg, Harmonic analysis and discontinuous groups in weakly symmetric Riemannian spaces with applications to Dirichlet series, J. Indian Math. Soc. 20 (1956), 47-87.

[16] R. Tsushima, A formula for the dimension of spaces of Siegel cusp forms of degree three, Amer. J. Math. 102 (1980), 937-977. 
[17] R. Tsushima, On the space of Siegel cusp forms of degree two, ibid. 104 (1982), 843-885.

MATHEMATISCHES INSTITUT DER UNIVERSITÄT FREIBURG

ALBERTSTR. 23 B

D-79104 FREIBURG I. BR., DEUTSCHLAND 